\title{
TRANSLATIONS IN CERT AIN GROUPS OF AFFINE MOTIONS
}

\author{
JOHN SCHEUNEMAN
}

ABSTRACT. The purpose of this article is to prove the conjecture of L. Auslander that every nilpotent group of affine motions of $\mathbf{R}^{n}$ that is simply transitive on $\mathrm{R}^{n}$ has a nontrivial translation in its center. The preliminary result that every such group is unipotent is of independent interest.

1. Introduction. In what follows, the points $x$ of $\mathbf{R}^{n}$ will be identified with column vectors $\left(\begin{array}{l}x \\ 1\end{array}\right)$. Affine motions are then $(n+1) \times(n+1)$ real matrices $\left(\begin{array}{cc}A & \nu \\ 0 & 1\end{array}\right)$ with $A$ a nonsingular $n \times n$ matrix, and the action on $\mathbf{R}^{n}$ is given by

$$
\left(\begin{array}{l}
x \\
1
\end{array}\right) \rightarrow\left(\begin{array}{ll}
A & v \\
0 & 1
\end{array}\right)\left(\begin{array}{l}
x \\
1
\end{array}\right)=\left(\begin{array}{c}
A x+v \\
1
\end{array}\right) .
$$

The matrix $A$ is called the homogeneous part, and the column vector $v$ the translation part of the affine motion $\left(\begin{array}{cc}A & v \\ 0 & 1\end{array}\right)$. Translations are the affine motions having the identity as their homogeneous part.

A group $G$ of transformations of a space $X$ is said to be simply transitive on $X$ if, given any two points $x$ and $y$ of $X$, there is a unique $g$ in $G$ such that $g x=y$. In such a case, the isotropy group at each point is the identity; the $G$-orbit of each point is all of $X$; the only element of $G$ with a fixed point is the identity. When $G$ is a group of affine motions of $\mathbf{R}^{n}$, $G$ is simply transitive on $\mathbf{R}^{n}$ if and only if, given any $x$ in $\mathbf{R}^{n}$, there is a unique element of $G$ having $x$ as translation part, and then the map taking an element of $G$ to its translation part is a bijection of $G$ onto $\mathbf{R}^{n}$. Such a $G$ is clearly a connected, simply connected Lie group.

L. Auslander noticed that in all the known examples (see [2]) of nilpotent simply transitive groups of affine motions of $\mathbf{R}^{n}$, there is a nontrivial translation in the center of the group, and conjectured that this is always the case. Here we verify this conjecture. The proof is carried out in two

Received by the editors November 29, 1973.

AMS (MOS) subject classification s (1970). Primary 22E 25; Secondary 17 B 30.

Key words and phrases. Simply transitive nilpotent group of affine motions, unipotent, translation. 
steps. First it is proved in $\$ 2$, using an argument of Auslander, that if $N$ is a nilpotent group of affine motions that is simply transitive on $\mathbf{R}^{n}$, then $N$ is unipotent. It follows that $N$ may be assumed to consist of upper triangular matrices with 1 's on the main diagonal, and our problem reduces to an analogous one about Lie algebras of upper triangular nilpotent matrices which is solved in $\$ 3$.

\section{Reduction of the problem.}

Theorem 1. If $N$ is a nilpotent group of affine motions of $\mathbf{R}^{n}$ that is simply transitive on $\mathbf{R}^{n}$, then $N$ is unipotent.

Proof. Let $h(N)$ be the group of homogeneous parts of elements of $N$. Then $h(N)$ is a homomorphic image of $N$, so it is nilpotent. Also, $h(N)$ is connected in the euclidean topology because it is the image of $\mathbf{R}^{n}$ under the continuous map $A$, where $A$ is defined by the bijection $x \rightarrow\left(\begin{array}{cc}A(x) & x \\ 0 & 1\end{array}\right)$ of $\mathbf{R}^{n}$ onto $N$.

Next let $G$ be the intersection of all the $n \times n$ complex matrix groups that contain $h(N)$ and are algebraic groups. Then $G$ is a nilpotent algebraic group (see $[1, \S 4]$ ). Furthermore, $G$ is connected in the Zariski topology, for in any case, the identity component of $G$ is an algebraic group and must contain $h(N)$ since $h(N)$ is connected in the euclidean topology and the cosets of the identity component of $G$ are disjoint. Therefore (see $[1, \$ 11]$ ) $G$ is a direct product $G=S \times U$ of algebraic groups, where $S$ is abelian and each element of $S$ is semisimple, and $U$ is unipotent. $S$ is diagonalizable (see $[1, \S 7]$ ), so there are subspaces $V_{1}, \ldots, V_{k}$ on each of which every element of $S$ acts as a scalar. Since $S$ is contained in the center of $G$, elements of $U$ preserve each $V_{i}$. Each $V_{i}$ therefore has a basis with respect to which each element of $U$ has an upper triangular unipotent matrix (see $[1, \$ 19])$. From this it follows that there is a basis of $\mathrm{C}^{n}$ with respect to which all elements of $G$ are simultaneously in block form

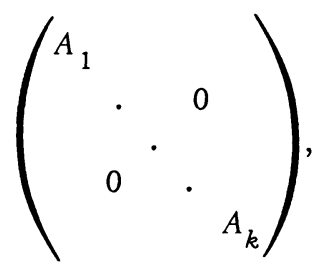

where each $A_{i}$ is upper triangular with equal diagonal entries.

The above reasoning shows that there is a complex matrix $T=\left(\begin{array}{ll}P & 0 \\ 0 & 1\end{array}\right)$ such that the elements of $T N T^{-1}$ are simultaneously in block form 


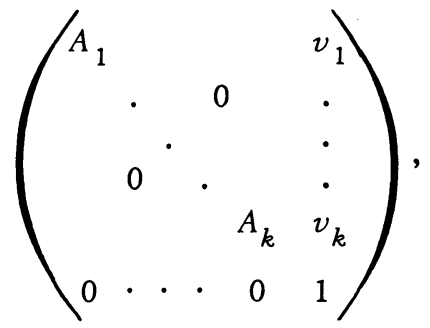

where, as above, each $A_{i}$ is upper triangular with constant diagonal.

Assume that some $X_{1}$ in $T N T^{-1}$ has an eigenvalue $\lambda \neq 1$ in the $i$ th block. Let $v_{i}$ be the $i$ th translation part of $X_{1}$; then conjugation of $T N T^{-1}$ by

$$
T_{1}=\left(\begin{array}{cccc}
\lambda I & & 0 \\
& & & \vdots \\
0 \ldots 0 & \lambda\left(A_{i}-I\right)^{-1} v_{i} & 0 \cdots 0 & 1
\end{array}\right)^{T}
$$

( $T$ denotes transpose) preserves the block structure and takes $X_{1}$ to a matrix $X$ having $i$ th translation part equal to zero.

Next observe that there is some $Y$ in $T_{1} T N T^{-1} T_{1}^{-1}$ which has as its $i$ th translation part a column vector whose bottom entry $x$ is nonzero. The reason for this is that the set of all translation parts of elements of $T_{1} T N T^{-1} T_{1}^{-1}$ is the image of $\mathbf{R}^{n}$ under a single complex affine motion of $\mathbf{C}^{n}$ onto $\mathbf{C}^{n}$. Accordingly, the complex rank of this image of $\mathbf{R}^{n}$ is $n$, and the existence of $Y$ follows.

Now calculate commutators $Y_{1}=X Y X^{-1} Y^{-1}, Y_{2}=X Y_{1} X^{-1} Y_{1}^{-1}$, etc. The triangular block shape of $X$ and $Y$ means that $X$ and $Y$ have submatrices

$$
\left(\begin{array}{ll}
\lambda & 0 \\
0 & 1
\end{array}\right) \text { and }\left(\begin{array}{cc}
\mu & x \\
0 & 1
\end{array}\right) \text {, }
$$

respectively (recall that $x \neq 0$ is the bottom entry of the $i$ th translation part of $Y$ ), and that $Y_{r}$ has a submatrix

$$
\left(\begin{array}{cc}
1 & (\lambda-1)^{r} x \\
0 & 1
\end{array}\right) .
$$

Therefore, none of the higher commutators $Y_{r}$ is the identity, which contradicts the assumption that $N$ (and hence each conjugate of $N$ ) is nilpotent. This proves Theorem 1.

Since $N$ is unipotent, there is a real matrix $T=\left(\begin{array}{ll}P & 0 \\ 0 & 1\end{array}\right)$ such that each element of $T N T^{-1}$ is upper triangular unipotent. Affine conjugation preserves translations, so it suffices to prove the existence of a central translation when $N$ is upper triangular. In this case, the Lie algebra of $N$ is 
an $n$-dimensional matrix Lie algebra consisting of $(n+1) \times(n+1)$ upper triangular nilpotent matrices $\left(\begin{array}{cc}A & v \\ 0 & 0\end{array}\right)$, where for each $v$ in $\mathbf{R}^{n}$ there is a unique element in the algebra having $v$ as its "translation part". The exponential of a nontrivial central element of the form $\left(\begin{array}{ll}0 & v \\ 0 & 0\end{array}\right)$ is a nontrivial central translation in $N$, so our problem reduces to one about matrix Lie algebras as above.

3. Conclusion. In this section, $L$ denotes an $n$-dimensional Lie algebra of $(n+1) \times(n+1)$ upper triangular nilpotent matrices $\left(\begin{array}{ll}A & v \\ 0 & 0\end{array}\right)$ whose "translation parts" $v$ make up all of $\mathbf{R}^{n}$. For future reference, notice that conjugation of $L$ by origin-preserving affine motions $\left(\begin{array}{ll}P & 0 \\ 0 & 1\end{array}\right)$ preserves central translations, and also preserves the property that translation parts make up all of $\mathbf{R}^{n}$. Therefore the existence of central translations in $L$ is established by

Theorem 2. Let L be a Lie algebra as above. Then there is some origin-preserving affine motion $T$ such that $T L T^{-1}$ is as above and contains

$$
\left(\begin{array}{cc}
0 & e_{1} \\
0 & 0
\end{array}\right), \text { where } e_{1}=\left(\begin{array}{l}
1 \\
0 \\
0
\end{array}\right) \text {. }
$$

Proof. The theorem is true if $L$ is one dimensional. Make the induction assumption and let $L$ be an $n$-dimensional algebra as above. Let $M$ be the subset of $L$ consisting of the elements whose translation part has bottom entry zero. Then because of the shape of the elements of $L, M$ is a subalgebra (in fact an ideal) of $L$, and because of the assumption about translation parts, $M$ has dimension $n-1$. Deleting the $n$th row and $n$th column (that is, the next to last row and column) of elements of $M$ gives a representation of $M$ as an algebra satisfying the induction hypothesis. It follows that there is some

$$
T=\left(\begin{array}{lll}
P & 0 & 0 \\
0 & 1 & 0 \\
0 & 0 & 1
\end{array}\right)
$$

'such that $L_{1}=T L T^{-1}$ is upper triangular and contains

$$
Z=\left(\begin{array}{lll}
0 & u & e_{1} \\
0 & 0 & 0 \\
0 & 0 & 0
\end{array}\right)
$$

If the vector $u$ is zero, we are through. Suppose that $u$ is not zero, and 
write $X$ for the element

$$
\left(\begin{array}{lll}
A & w & 0 \\
0 & 0 & 1 \\
0 & 0 & 0
\end{array}\right)
$$

of $L_{1}$. Let $Z_{1}=[X, Z], Z_{2}=\left[X, Z_{1}\right]$, etc. Then we have

$$
Z_{r}=\left(\begin{array}{ccc}
0 & A^{r} u & -r A^{r-1} u \\
0 & 0 & 0 \\
0 & 0 & 0
\end{array}\right),
$$

so that since $A$ is nilpotent one of these is a nonzero element

$$
W=\left(\begin{array}{lll}
0 & 0 & t \\
0 & 0 & 0 \\
0 & 0 & 0
\end{array}\right)
$$

in $L_{1}$. The set of all such elements of $L_{1}$ is an ideal $J$, and since $L_{1}$ is nilpotent, the sequence of ideals

$$
J \supseteq\left[L_{1}, J\right] \supseteq\left[L_{1},\left[L_{1}, J\right]\right] \cdots
$$

descends to zero. Hence some $W$, as above, is in the center of $L_{1}$. If $t$ is a scalar multiple of $e_{1}$, we are through. Otherwise, write

$$
t=\left(\begin{array}{llll}
t_{1} \cdots t_{k} & 0 \cdots 0
\end{array}\right)^{T},
$$

where $T$ denotes the transpose, $t_{k} \neq 0$ and $k>1$. We may take $t_{k}=1$. Then it is easy to show (induction) that there is a nonsingular upper triangular matrix $Q$ such that $Q t=e_{k}=\left(\begin{array}{llll}0.0 & 1 & 0 \ldots 0\end{array}\right)^{T}$. Then the algebra

$$
L_{2}=\left(\begin{array}{ll}
Q & 0 \\
0 & 1
\end{array}\right) L_{1}\left(\begin{array}{ll}
Q & 0 \\
0 & 1
\end{array}\right)^{-1}
$$

is upper triangular and contains a central element

$$
W_{1}=\left(\begin{array}{cc}
0 & e_{k} \\
0 & 0
\end{array}\right) \text {. }
$$

Since $W_{1}$ is central, the $k$ th column of every element of $L_{2}$ is zero. Let $L_{3}$ be the conjugate of $L_{2}$ gotten by interchanging the first and $k$ th rows and also the first and $k$ th columns of elements of $L_{2}$. Then $L_{3}$ contains

$$
\left(\begin{array}{cc}
0 & e_{1} \\
0 & 0
\end{array}\right)
$$


and each element of $L_{3}$ has first column zero, so each may be written in the form

$$
\left(\begin{array}{lll}
0 & a & c \\
0 & A & b \\
0 & 0 & 0
\end{array}\right)
$$

The map

$$
f:\left(\begin{array}{lll}
0 & a & c \\
0 & A & b \\
0 & 0 & 0
\end{array}\right) \rightarrow A
$$

is a Lie algebra homomorphism and $f\left(L_{3}\right)$ is a Lie algebra of nilpotent $(n-1) \times(n-1)$ matrices, so there is a matrix $R$ such that $R f\left(L_{3}\right) R^{-1}$ is upper triangular. Hence

is upper triangular and contains

$$
\left(\begin{array}{lll}
1 & 0 & 0 \\
0 & R & 0 \\
0 & 0 & 1
\end{array}\right) L_{3}\left(\begin{array}{lll}
1 & 0 & 0 \\
0 & R & 0 \\
0 & 0 & 1
\end{array}\right)^{-1}
$$

$$
\left(\begin{array}{lll}
0 & 0 & e_{1} \\
0 & 0 & 0 \\
0 & 0 & 0
\end{array}\right)
$$

This proves Theorem 2 and establishes the conjecture of Auslander.

\section{REFERENCES}

1. A. Borel, Groupes linéaires algébriques, Ann. of Math. (2) 64 (1956), 20-82, MR 19, 1195.

2. J. Scheuneman, Affine structures on three-step nilpotent Lie algebras, Proc. Amer. Math. Soc. 46 (1974), 451-454.

24 SOUTH DUTCHER STREET, IRVINGTON, NEW YORK 10533 